\title{
Real time trajectory prediction for collision risk estimation between vehicles
}

\author{
Samer Ammoun \\ Imara project \\ Inria Rocquencourt \\ France \\ samer.ammoun@mines-paris.org
}

\author{
Fawzi Nashashibi \\ Imara Project, Inria Rocquencourt \\ \& Robotics center, Ecole des Mines de Paris, \\ France \\ fawzi.nashashibi@inria.fr
}

\begin{abstract}
In this paper, we present our approach for collision risk estimation between vehicles. The vehicles are equipped with GPS receivers and communication devices. Our approach consists on using the knowledge given trough communication tool to predict the trajectories of the surrounding vehicles. Based on these trajectories, we identify the configurations of the collisions between vehicles. The risk is calculated using several indicators that are reflecting not only the possible collisions but also the dangerousness of these collisions. Our algorithm is tested on crossroads using scenarios involving real prototypes producing realistic scenarios.
\end{abstract}

Keywords-Trajectory prediction, risk assessment, cooperative driving, vehicle dynamic model.

\section{INTRODUCTION}

The reduction of the amount of accidents on the roads is still a priority in the transportation domain in general and particularly in the ITS ${ }^{1}$ domain. In fact, less accidents means more secure, more efficient and stress less driving. Actually, vehicles are considered as the main source of danger on roads since they are generally the major actors in the accidents. For these reasons, our work is aiming to give the vehicle some intelligence in order to be able to reduce the risk of accidents. As a first step, the vehicle gets the capacity to sense its environments via its own sensors. In the second step, the vehicle will be able to estimate and to deal with the risk incurred during the navigation. In this paper, we are focusing on the vehicle-vehicle accident cases. The vehicle-static objects accidents are considered as trivial and implicit cases for this algorithm. For the case of vehiclevulnerable objects ${ }^{2}$, our method is still valid with a need to propose a model of displacement for the vulnerable objects.

Regarding the possible help that the new technologies may offer to the driver, we consider that the nature of the risk is very related to the degree of the driver's responsibility. In other words, it depends on the degree of automation of the vehicle. We identify three levels to deal with the risk corresponding to the degree of automation of the vehicle :

1) Driver assistance system : in this case, the driver has a full control of the vehicle. The vehicle's intelligence

\footnotetext{
${ }^{1}$ Intelligent Transportation Systems

${ }^{2}$ as pedestrian or bicycles
}

consists in the elaboration of adapted recommendations to the driver in order to help him to deal with risky situations. Some active safety features are now taking place into the vehicles but the driver is still the unique master of its own vehicle. The role of the vehicle is to sense the environment and to analyze the information about the neighborhood (no matter if the information is processed and analyzed on board the vehicle or exchanged via communication). The vehicle will perform periodically a prediction of the future situation of its neighborhood and detect any future risky configuration. The complexity of the prediction is related to the fact that the prediction is computed based on the recent information about the environment. The prediction does not take into consideration the next behavior of the driver who has the total liberty to change the future trajectory of the vehicle using the available actuators (brake pedal, steering wheel, gear and accelerator pedal). Our presented work is situated at this level of risk estimation.

2) Fully automated driving with standalone planning : in this case, the driving is fully automated and no role is reserved for the driver. The role of the vehicle is to sense its environment, to perform a planning of the next trajectory and to supervise the right execution of this path. The goal of the motion planner is to reach a final position with some constraints: collision free, lane keeping, etc. Regarding that the planning is performed locally, each vehicle will continue to estimate any possible collision with the trajectories of the neighbor vehicles.

3) Cooperative planning: here the vehicles, within the same neighborhood, are generating cooperatively riskfree trajectories. The navigation is then 100 percent safe. Because some hazardous situations are still possible, the vehicles keep running a collision detection algorithm.

In this paper, we will present our approach for performing risk estimation related to collisions between non-automated vehicles. In the first section, we will present an overview of our approach. In the following sections, we will detail 
different parts of our algorithm. Before concluding, we will present some results of our algorithm experimented on real scenarios.

\section{GLOBAL APPROACH FOR RISK ASSESSMENT}

Our approach consists of reducing the risk of collision through four sequential actions : environment modeling, trajectory prediction, collision detection and risk management. these steps are illustrated on the block diagram of the figure 1 .

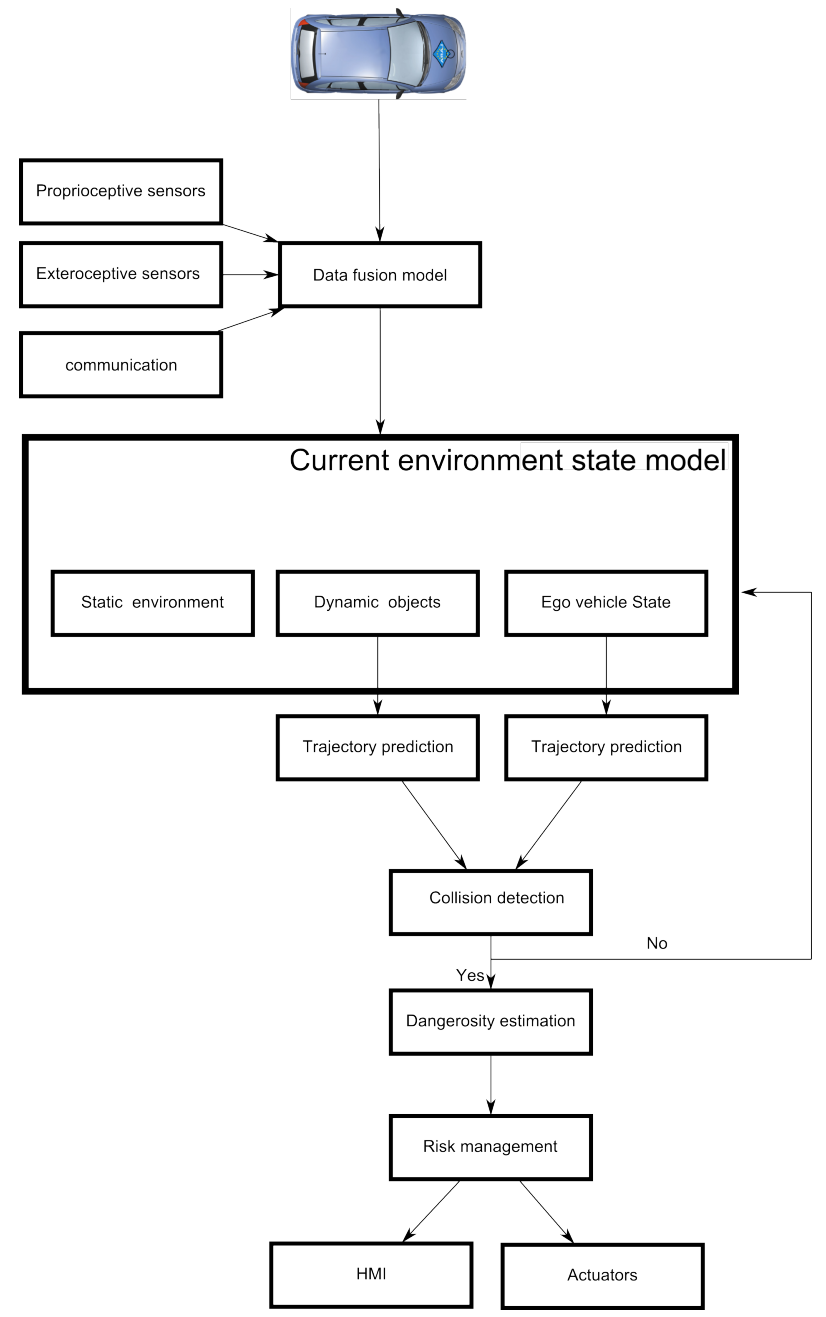

Figure 1. A block diagram summarizing the risk assessment steps

In this work, we suppose that we dispose of the current state of the vehicle environment. In fact, modeling the environment of navigation consists on proposing a representation of the surrounding environment. This model should offer not only the static geometry of the roads but also the positions and the attributes of the dynamic objects on (and next to) the roads.
This representation is provided to the vehicle using (separately or combined) two different approaches regarding the source of information :

- Standalone approach : each vehicle is responsible of building its own environment map based on the output of its internal and perception sensors. The drawback of this method is that the knowledge of the vehicle is limited quantitatively by the range of its sensors and qualitatively by the precision and the richness of the delivered information. In this approach, we are relying completely on the detection and the classification algorithms to give a description of the environment (static or mobile objects, types of vehicles, pedestrians, etc.)

- Cooperative approach : based on inter-vehicle communication, the vehicles are able to exchange information about its respective environment. Each vehicle is thus receiving an accurate and rich information from the surrounding vehicles. The drawback of this method is that it is depending on the number of vehicles equipped with communication devices. With this method, a nonequipped vehicle is totally invisible to other vehicle.

At least during the period of deployment of the communication devices in vehicles, it is important to combine the two methods to take advantages of both approaches.

Based on this local map and in order to estimate any possible risky configurations, we proceed by projecting the current environment state model into the future. This prediction step is considered as the most delicate step in our approach. In fact, we proceed by predicting the behavior of each vehicle accounting on the current inputs. Nonetheless, we do not attempt to predict in any case the future behavior of the driver. In fact, any change in the driver's behavior is going to potentially influence the inputs of the system and consequently the future trajectory of the vehicle.

Each environment state model (and naturally the predicted trajectories) is taking into account the errors and the imprecisions on the positions and on the attributes of the different entities in the scene. These errors are due to the errors on the sensors outputs and/or the errors of detection and classification algorithms.

Once predicted for the ego vehicle and for each neighboring vehicle, the future trajectories are utilized to compute the possible collision between each couple of trajectories. This approach considers that the risk of collision is independent between each couple of vehicles. Obviously, the problem is more complex since we should take into consideration the whole situation in order to estimate this risk. In our application, we made the choice of exploiting the collision detection not for path planning 
but only for collision prevention.

Based on geometric and dynamic models of the vehicle, the dangerousness of the collision is estimated using several risk indicators.

In order to avoid accidents, two actions are possible with different degrees of implication in the decision and therefore with differences from the responsibility and legacy points of view.

- The informative action : the ADAS system will inform the driver about the dangerousness of the situation and potentially propose some maneuvers to reduce the risk. This information is delivered through an appropriate HMI. In this paper, we do limit our intervention on this level.

- The active behavior : in this system, the vehicle takes the initiative and operates on the actuators to realize the adequate to perform collision avoidance

In the next sections, we will describe in detail each part of this algorithm.

\section{TRAJECTORY PREDICTION}

A trajectory is not only a list of geographic positions of the vehicle. It is a spatio-temporal representation of the displacement of the vehicle. In other terms, it is a dynamic representation of the path. The trajectory prediction is considered as a key step in our methodology. Taking into consideration that it is very difficult to predict the human behavior, we assume that the aim of our approach is to predict at the time $t_{0}$ the future displacement of the vehicle during the time slice $\left[t_{0}, t_{0}+h\right]$ in case that the driver maintains the same driving profile. $h$ is prediction period in seconds.

Whatever the prediction algorithm is, the period between the incoming of new inputs on the actuators of the vehicle and the integration of these inputs into our prediction model will always be considered as a period of uncertainty for the risk estimator. For this reason, the faster is the algorithm of prediction the more reactive is the system and the shorter is this period of uncertainty.

The quality of the prediction can be estimated by measuring the errors committed between the predicted and the real trajectories of the vehicle.

In the next sections, we will expose the most used methods to compute the future trajectories of vehicles.

\section{A. Geometric approach}

Based on the fact that the roads are designed with specific geometric models, the trajectory of the vehicle can be modeled with geometric features since it is constrained to follow the roads. Some research works propose to use a polynomial representation of the trajectories. First, third and fifth degree polynomials are the most used regarding the complexity of the speed and acceleration models [2]. Others are using one or more clotoids to model the trajectory taking into account the continuity and the derivability of the entire profile [1]. The advantage of this shape-based trajectory generation is the computation facilities that assure a reduced time of estimation. It is also used to predict some trajectory before the beginning of the maneuver [4]. The main drawback is that this predictor is not based on any model of the vehicle. Moreover, the shape of the roads is not able to determine totally the behavior of the vehicle especially on some parts of the roads where there are many possibilities as on crossroads.

\section{B. Dynamic approach}

In this approach, the model tends to reproduce the dynamic behavior of the vehicle. Many models exists regarding the degree of complexity of the representation. Between the complicated models (like Ackeremann model) and the simplest one (as the bicycle model), the shapes of the equations are different regarding the inputs of the system. Different levels of inputs can be considered as the acceleration, speed, yaw angle, steering wheel rate, brake and acceleration pedal pressure [3], gear state etc. In spite of the complexity of this method, the main advantage is that the future trajectory is computed based on a vehicle model. The position, speed and acceleration are direct outputs of the predictor.

We choose to use the bicycle dynamic model for its simplicity. We integrate the model using a Kalman linear filter with the positions, the speed and the acceleration as inputs.

The path prediction is performed using a linear Kalman filter. By using this recursive filter, we will be able to:

- Filter the noisy GPS position.

- Estimate the position of the vehicle between two outputs of the GPS receiver.

- Estimate a horizon of displacement regarding the motion model of the vehicle.

- Estimate the positioning errors: this filter determines the ellipse of uncertainty which is the ellipse where the vehicle had the maximum probability to exist.

In the prediction step, the state vector $X$ and the covariance matrix $P$ are estimated in each iteration using the equations:

$$
\begin{aligned}
& \hat{X}_{k / k-1}=A_{k-1} \hat{X}_{k-1 / k-1}+B_{k-1} u_{k-1} \\
& P_{X_{k / k-1}}=A_{k-1} P_{X_{k-1 / k-1}} A_{k-1}^{t}+R_{v_{k-1}}
\end{aligned}
$$

In our case, $X$ is expressed as follow

$$
\hat{X}=\left(\begin{array}{c}
x \\
y \\
V_{x} \\
V_{y} \\
a_{x} \\
a_{y}
\end{array}\right)
$$


The prediction matrix $A$ is :

$$
\left(\begin{array}{cccccc}
1 & 0 & \Delta T & 0 & 0 & 0 \\
0 & 1 & 0 & \Delta T & 0 & 0 \\
0 & 0 & 1 & 0 & \Delta T & 0 \\
0 & 0 & 0 & 1 & 0 & \Delta T \\
0 & 0 & 0 & 0 & 1 & 0 \\
0 & 0 & 0 & 0 & 0 & 1
\end{array}\right)
$$

In our system, we don't have a control input $u$ so no need for the transformation matrix $B . P$ is the error covariance matrix and $R_{v}$ is the covariance of the process noise which is assumed to be a zero mean normal distribution.

The correction state is performed each time we received a new GPS position. The observation vector $Z$ is equal to:

$$
Z_{k}=\left(\begin{array}{c}
x \\
y \\
V_{x} \\
V_{k}
\end{array}\right)
$$

$Z$ is linked to the state vector through the following equation:

$$
Z_{k}=C_{k} X_{k}
$$

Where

$$
C_{k}=\left(\begin{array}{cccccc}
1 & 0 & 0 & 0 & 0 & 0 \\
0 & 1 & 0 & 0 & 0 & 0 \\
0 & 0 & 1 & 0 & 0 & 0 \\
0 & 0 & 0 & 1 & 0 & 0
\end{array}\right)
$$

is the observation matrix.

The optimal Kalman gain is then calculated using this equation:

$$
K_{k}=P_{X_{k / k-1}} C_{k}^{t}\left[C_{k} P_{X_{k / k-1}} C_{k}^{t}+R_{n}\right]^{-1}
$$

And regarding the calculated gain, we update the state vector $X$ and the error covariance matrix $P$ :

$$
\begin{gathered}
\hat{X}_{k / k}=\hat{X}_{k / k-1}+K_{k}\left(Z_{k}-C_{k} \hat{X}_{k / k-1}\right) \\
P_{k / k}=\left(I-K_{k} C_{k}\right) \times P_{k / k-1}
\end{gathered}
$$

$I$ is the identity matrix.

To be able to use this filter, we are assuming that the observation noise is a zero mean Gaussian white noise.

\section{Positioning UnCERTAinty AND VEHICLE BOUNDARIES}

It is very common that a GPS based representation of the position of the vehicle is modeled using an elliptic shape due to the probabilistic uncertainty model. This shape is representing the probability of presence. Thus, the collision between vehicles is performed by calculating the intersection between the corresponding ellipses.

Because the intersection calculation between ellipses is not analytically easy, we use a method commonly utilized in the 3D modeling domain. This method consists on modeling the ellipse by a series of aligned circles as shown in the figure 2. The detection of the collision between the vehicles is therefore performed by calculating the distances between the respective circles of each vehicle. The use of this representation is also useful for the determination of risk indicators as explained in next paragraph. A collision is detected if one of the circles of the vehicle is intersecting at least one of the other vehicles circles as illustrated on the figure 2 .

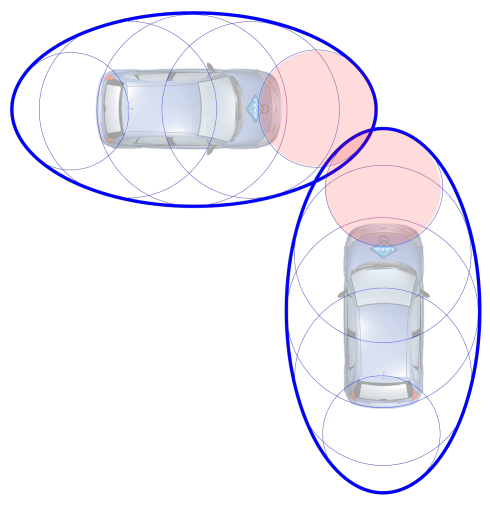

Figure 2. The collision detection is performed by detecting the collision between the circles around each vehicle

\section{RISK INDICATORS}

In case we detect a possible collision, the main parameter to study is the time that remains before the first impact between the vehicles. This time is important because it delivers the duration in witch the driver can act to avoid the collision or minimize its dangerousness. This parameter is called time to impact TTC. It is calculated as the duration between the current time and the instant of the first impact between the vehicle if the respective vehicles are keeping the current speed vectors. In our study, we compare this TTC to the duration of 2 seconds which is commonly recognize as the reaction time of the driver $(1 \mathrm{sec})$ and of the vehicle (1 sec). However, this parameter does not give any indication about the confidence of this detection or the possible way to minimize the risk related to this accident.

For these reasons, we propose three more kinds of indicators. Those parameters should be analyzed together in order to constitute a clear view of the possible collision :

- The number of circles implicated in the collision : This parameter give us an idea about the dangerousness of the collision. In fact, a collision that involves a larger number of circles is considered more dangerous.

- The duration of the predicted collision : this indicator gives an idea about the robustness of the estimation. The longer is this time the more sure is the collision. 
- The configuration of the collision : this parameter corresponds to the position of the circles implicated in the detected collision. This parameter is useful to propose a safe method to minimize the risk and to customize the avoidance maneuver regarding the configuration during the predicted collision.

For the moment, the TTC is still the main risk indicator. We aims to propose a risk that combines at least the TTC, the number of circles and the time of the collision in one risk function. The configuration of the collision is only useful in the case that we propose maneuver to avoid the collision.

\section{EXPERIMENTATION}

Many experiences were led with our system using the fleet of vehicles $\mathrm{LaRA}^{3}$. In this paper, we are presenting the tests performed on crossroads. The two vehicles LaRA1 and LaRA2 are cooperating via communication by exchanging their positions and their speed. The experience on real vehicles is conducted in the Velizy suburb of Paris. The two vehicles are equipped with similarly with a Trimble Ag332 GPS receivers and a Dlink 2100 Wifi access point. The frequency of the communication frames was harmonized with the GPS data frequency $(10 \mathrm{~Hz})$. In this experience, we are not using any high level protocol of communication. In fact we are using the libpcap library [5] to address directly the communication device. For more information about the communication procedure, please refer to [6]

Each vehicle LaRA is receiving the positions and the speed of the other vehicle. It is performing a prediction of its own trajectory and of the trajectory of distant vehicle. The figure 3 presents the trajectories of both vehicles as predicted on LaRA2 for a temporal horizon of $10 \mathrm{sec}$. The blue (resp. the red) ellipses present the positions of LaRA1 (resp. LaRA2).

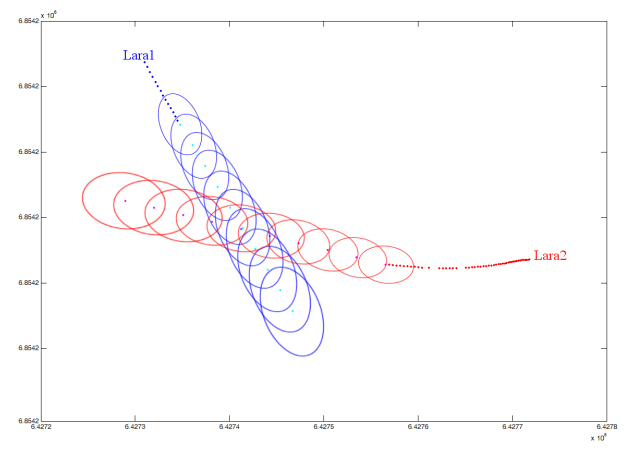

Figure 3. The trajectory of LaRA1 (blue) and LaRA2(red) during the experience. The estimated trajectories is represented by a series of uncertainty ellipses

\footnotetext{
${ }^{3}$ LaRA : La Route Automatise (The automated road in english) is a joint research unit between the Robotics center of the Ecole des Mines de Paris and the Imara project of the Inria Rocquencourt
}

During this experience, the vehicle were simulating a non controlled crossroads or red light violation situation. Both vehicles were approaching the crossroad zone without taking in consideration the presence of the other vehicle. The figure 4 presents respectively the TTC, the period of the estimated collision and the evolution of the percentage of circles implicated in the collision at the time TTC.
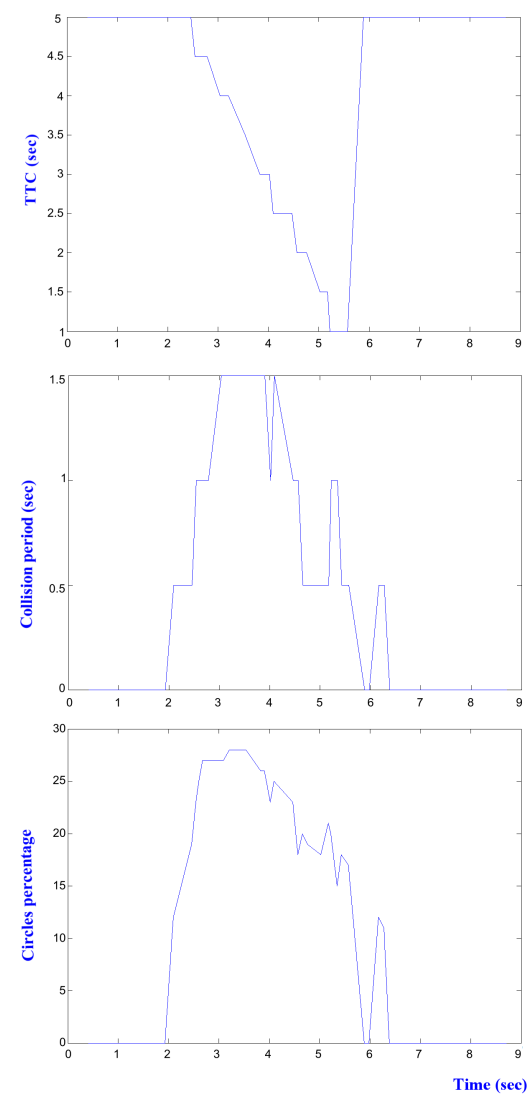

Figure 4. From top to bottom: the time to collision, the duration of the collision and the percentage of implicated circles during the experience of roads crossing

The analysis of these graphs shows that during this test, the time to collision is getting smaller as the vehicles are approaching the crossroad which means that the collision was becoming more unavoidable. At the same time, the period of collision is increasing; this means that the certainty of the estimation of the collision is getting higher. The percentage of circles involved in the collision is also increasing which means that the collision will be more and more dangerous. During this test, we did not go intentionally to the real collision, one of the vehicles brakes to let the other vehicle pass. This behavior is illustrated on the graph by the reduction of the dangerousness and the probability of the collision. Figure 5 shows an example of human machine interface that can be offered to the driver. On the left, we can 
see the dynamic map of the environment with the position of the local vehicle LaRA1 as obtained from the local GPS and the position of the distant vehicle LaRA2 as received by communication. On the same map, we can also see the predicted trajectories of both vehicles as estimated in LaRA1. On the right side, the risk indicator (associated only to the TTC) is presented by a 10 levels graphical indicator. On top of the HMI, the video is taken from LaRA1 during the experience.

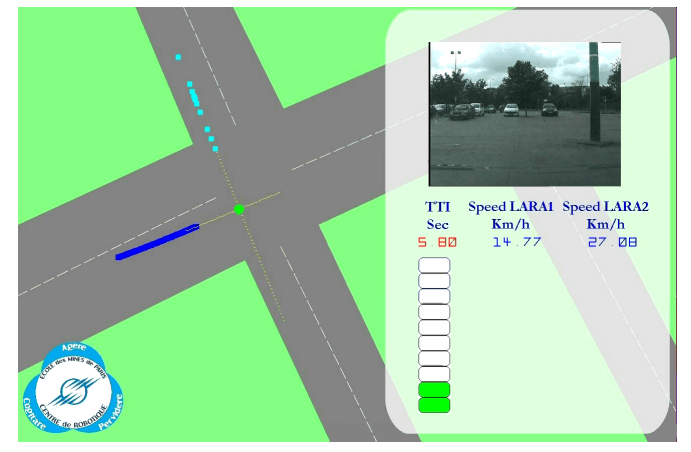

Figure 5. A prototype of HMI to present to the driver

This algorithm is running in real time on both vehicles. One of the main difficulties that we met was the communication signal loss in a no-line of sight configuration (typically on crossroads). This loss constrains the time to prevent the driver, reduces the change to anticipate the risk and make our algorithm more sensitive to the GPS error. Equipping the central part of the crossroads by communication relay could be the solution of this problem.

\section{CONCLUSION}

In this paper, we have presented our approach for risk assessment between vehicles. Our approach is based on predicting the future trajectory of each vehicle. The collision is detected using a special representation of the vehicle form with regard to the GPS errors. The enhancement of the risk estimator will be performed by enhancing the quality of each of his sequential parts. One of the main axes of enhancements is to propose an accurate model of trajectory prediction by proposing an accurate dynamic model of the vehicle and respectively more complex equations for the Kalman filtering. In this paper, we have also presented many risk indicators and we are working currently on merging these indicators in an unique function which describes the dangerousness of the accidents. The studied scenario is representing one risky situation on crossroads. The next step will be to study more complex behavior on crossroads like left(resp. right) turn across path.

\section{ACKNOWLEDGMENT}

This work was conducted within the research project INTERSAFE-2. INTERSAFE-2 is part of the 7th Framework Programme, funded by the European Commission. The partners of INTERSAFE-2 thank the European Commission for supporting the work of this project.

\section{REFERENCES}

[1] Accurate estimation of forward path geometry using twoclothoid road model, Khosla, D., Intelligent Vehicle Symposium, 2002. IEEE, Volume 1, Issue , 17-21 June 2002, Page(s): 154 - 159 vol.1

[2] Fast lane changing computations using polynomials, $\mathrm{Pa}-$ padimitriou, I. Tomizuka, M., Proceeding of the 2003 American Control Conference, 4-6 June 2003, Volume: 1, On page(s): 48- 53 vol.1

[3] Cooperative decentralized intersection collision avoidance using extended Kalman filtering, Ashil Sayyed Farahmand, Thesis submitted to the faculty of the Virginia Polytechnic Institute and State University, December 5, 2008

[4] An analysis of the lane changing manœuvre on roads : the contribution of inter-vehicle cooperation via communication, Ammoun, S. Nashashibi, F. Laurgeau, C., IEEE Intelligent Vehicles Symposium, 13-15 June 2007, Istanbul, On page(s): 1095-1100

[5] http://www.winpcap.org/

[6] Crossroads risk assessment using GPS and Inter-vehicle communications, Ammoun S., Nashashibi F. and Laurgeau C., In the Journal of IET International Journal on Intelligent Transport Systems. Paper number / Manuscript ID: ITS-20060076 